\title{
PERFORMANCE ANALYSIS OF A DYNAMIC CHANNEL ALLOCATION TECHNIQUE FOR TERRESTRIAL AND SATELLITE MOBIL CELLULAR NETWORKS
}

\author{
E. Del Re, R. Fantacci, G. Giambene \\ Universila di Firenze - Dipartimento di Ingegneria Elettronica \\ Via S. Marta, 350139 Firenze ITALY \\ Phone: +3955 4796285, Fax: +3955 494569
}

\begin{abstract}
This paper deals with an efficient Dynamic Channel Allocation (DCA) technique suitable for applications in boih terrestrial and satellite cellular networks. A cost function is defined to allow an optimum selection of the channel to be allocated on demand. The performance of the novel DCA technique in terms of blocking probability has been derived by simulations. The obtained results are compared with those achieved by a Fixed Channel Allocation (FCA) technique to show a better behaviour.
\end{abstract}

\section{INTRODUCTION}

The rapid growth in the demand for personal communications has led to the need of an intense research and developement efforts towards a new generation of Personal Communication Systems (PCSs). Any future PCS will integrate different services to provide data and phone communications via a multimedia portable terminal. The challenge is to build a Personal Communication Network (PCN) which allows the integration of PCSs with fixed Broadband Integrated Service Digital Networks (B - ISDNs).

This paper is focused on a PCN which is mainly devoted to phone communications in a mobile users environment (mobile communication system). The major issue here is to find a way to satisty the large demand consistent with limited bandwidth and high-quality service.

Work carted ous under the financial suppon of ASI and MURST. 0-7803-0917-0/93\$03.00 ○ 1993 IEEE
A limiting factor is the co-channel interference due to simultaneous use of the same channel in different spatial cells. Considering a mobile communication system with cellular coverage given by equal-size hexagonal cells, in order to reduce the co-channel interference, a same channel cannot be simultaneously used in cells whose centers are distant less than the reuse distance, D [1]. The derivation of $D$ depends on several factors including particular multiple access technique and modulation scheme used, mobile environment and acceptable voice quality.

The performance of a mobile communication system is increased by a better utilization of available channels. Referring to the O.S.I. network model, three are the layers on which we can operate to achieve this goal:

- Physical layer. we must proceed in cells splitting, keeping unvaried their capacity, so that the quantity of traffic carried on by the network increases $\left(\propto 1 / R^{2}\right.$, with $R$ denoting the hexagonal cells side).

- Data link layer. we must choose the multiaccess technique which is able to grant the available spectrum to the utmost possible number of simultaneous users .

- Network tayer. a suitable Dynamic Channel Allocation (DCA) technique can be used to allow a better utilization of network resources [2] [3].

In this paper we focus on the network layer. In particular an efficient DCA technique suitable for application in a mobile communication system is presented.

Differently from a Fixed Assignment (FCA) strategy, with the DCA proposed in this paper, each cell has no channel permanently allocated to it. Whenever a new call attempt arrives in a cell, the associated Base Station (BS) asks the Mobile Switching Center (MSC) for allocation of a new 
channel. The channel to be assigned is selected on the basis of a suitable cost function depending on the reuse distance and number of cells just using each available channel. The MSC decides, on a call-by-call basis, the channel to be allocated by exhaustive search for the minimum cost channel. In addition to this, an efficient channel rearrangement lechnique has been also considered whenever a call termination or handover occurs in a cell [4].

The performance of the DCA technique under consideration has been derived by considering that:

- the maximum number of available channels is equal to $\mathrm{M}$;

- calls duration is random and exponentially distributed with expected value equal to $T_{m}$;

- blocked calls are cleared;

- the offered traffic per cell is Poisson with the same mean arrival rate $\lambda$ in all the cells.

This paper has the following organization: Sect. II deals with a novel efficient DCA technique; in Sect. III handover and user mobility are discussed; finally, in Sect. IV simulation results concerning the performance of the proposed DCA technique in terms of call blocking probability are presented and compared with those obtained in the case of a FCA technique.

\section{DYNAMIC CHANNEL ALLOCATION TECHNIQUE}

In this section a novel (DCA) technique is presented. The proposed DCA technique achieves an efficient spectrum utilization by allowing the use of each of the $\mathrm{M}$ channels of the network in any cell on the condition that the constraint on $D$ is respected. We remark that in a mobile communication system $D$ represents the minimum distance between cells for which the use of the same channel gives rise to an acceptable cochannel interference level.

Let us consider a particular cell $x$ and denote with $l(x)$ the set of interfering cells with $x$, i.e. those cells for which it is not permitted to allocate any channel just used in $x$.

Note that also the vice-versa is valid. Fig. 1 shows the cells $\in I(x)$ for the case $D=\sqrt{21} R$, with $A$ denoting the hexagonal cells side [1]. From above, it is evident that a channel $i$ is available for allocation to the cell $x$ if it is not used either in $x$ or in $I(x)$.

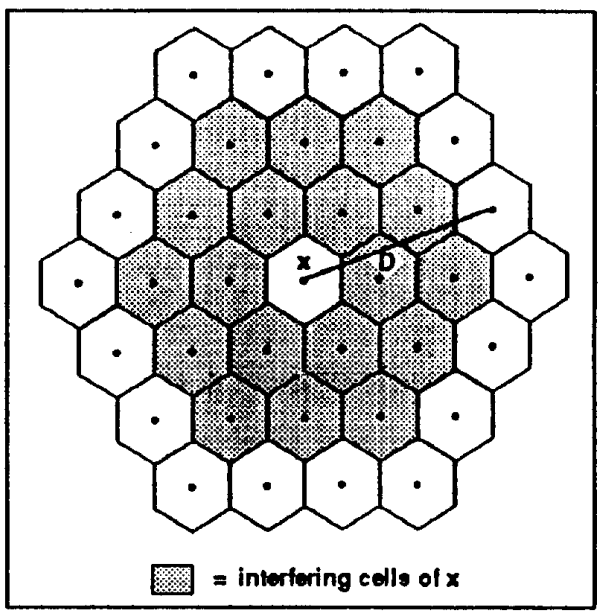

Fig. 1: cell $x$ and interfering cell of $x$, for reuse distance $D=\sqrt{ } 21 \mathrm{R}$.

Let us denote with $\Lambda(x)$ the set of available channels for the cell $x$ at the call arrival instant.

For each channel $i \in \Lambda(x)$, we define the following cost function:

$$
C_{x}(i) \Delta \sum_{k \in i(x)}\left\{u_{k}(1)\right\}
$$

where:

$$
u_{k}(1)= \begin{cases}1, & \| \in \Lambda(k) \\ 0, & \text { otherwise }\end{cases}
$$

Whenever a new call arrives in $x$, if $\Lambda(x) \neq \varnothing$, the channel $i$ which verifies the following condition:

$$
i^{*}: C_{x}\left(i^{*}\right)=\min _{1 \in A(x)}\left\{C_{x}(1)\right\}
$$

is temporarily assigned to $x$. Note that the selection of the channel $i$ according to (3), permits to minimize the number of cells $\in I(x)$ in which the use of the same channel $i$ will be locked and therefore to increase the spectrum utilization.

li more channels verify (3) a random choice is performed.

In the case of $\Lambda(x)=\varnothing$ (i.e. no channel can be temporarily assigned to the cell $x$ ), the call attempt is blocked and lost. The updating of $\Lambda(x)$ and therefore the evaluation of the cost $C_{x}(i)$ for any channel $i \in \Lambda(x)$ is performed on the basis of tables. 
These tables take into account the channels availability in all the cells of the network in the case of centralized implementation of the DCA or only in cells $\in\{x\} \cup I(x)$ in the case of a distributed implementation.

In order to obtain a better performance, whenever we have a call termination in a cell, the DCA technique performs a channel rearrangemen! to de-allocate the channel which becomes available in the greatest number of cells $\in I(x)$.

The final result is that the DCA technique allows an optimum channel allocation to the cells on demand i.e. an efficient spectrum utilization.

\section{HANDOVER AND USERS MOBILITY}

In mobile communication systems the handover procedure has a significant impact on the system performance, in particular when microcells are used. Handover can occur in the time interval during which the ratio of the power levels received from the base station (BS) of the current cell (source cell) and the BS of a neighbouring cell (destination cell of the mobile) is within two appropriate thresholds [5].

The handover procedure gives rise to a call termination in the source cell jointly with a new call arrival in the destination cell.

Different handover policies have been recently proposed. Some of them prioritize handovers at the expense of an increased call blocking probability. The prioritization schemes are motivated by the fact that from the Mobile Subscriber (MS) point of view, forced termination of an ongoing call is greatly less desirable than blocking a new call attempt.

This paper deals with handover policy which manages handover requests in exactly the same manner as new call attempts. However, it will be shown by computer simulation that the novel DCA lechnique permits indeed to privilege the handovers service with respect to the one of new call attempts to achieve a better quality of service.

To investigate the influence of the handover procedure on the performance of the mobile communication system under consideration, we must carefully account for the MSs mobility. Let us assume that:

- every user runs through a cell with a uniformly distributed distance from 0 to $2 \mathrm{R}$, where $R$ is the cells side;
- every user travels in a cell with a constant velocity, uniformly distributed from 0 to $V_{\text {max }}$;

- every adjacent cell has an equal probability to be the destination cell for the mobile.

Moreover, we define the following parameter:

$$
\alpha \Delta \frac{2 R}{V_{\max } T_{m}}
$$

to characterize the user mobility ( $\alpha$ decreases when users mobility increases). The probability density function (p.d.f.) of the time interval during which a $M S$ is within the source cell from the call beginning instant, $t_{m c}$ can be derived as:

$$
\text { p.d.f. } t_{m a}(t)=\left\{\begin{array}{l}
\frac{V_{\max }}{4 R}, \text { for } 0 \leq t \leq \alpha T_{m} \\
\frac{R}{V_{\max } t^{2}}, \text { for } t>\alpha T_{m}
\end{array}\right.
$$

Let $t_{d}$ be the call duration time (exponentially distributed with expected value equal to $T_{m}$ ). Note that a handover procedure occurs whenever $t_{m c}<$ $t_{d}$. Hence, the handover probability, $P_{h}$, can be given by:

$$
P_{h}=\int_{0}^{\infty} \operatorname{Prob} .\left(\zeta_{d}>t \mid t_{m c}=t\right) \text { p.d.t. } f_{h o}(t) d t
$$

From (6), through some algebraic manipulations we have:

$$
P_{h}=1-\left\{\frac{2 \alpha-1-\theta^{-\alpha}(\alpha-1)}{2 \alpha}+\frac{\alpha}{2} \int_{\alpha}^{-} \frac{\theta^{-x}}{x} d x\right\}
$$

Note that if $\alpha$ approaches $0(\infty), P_{h}$ approaches 1 (0).

The probability that a call in progress in a cell may have a handover to an adjacent cell is independent from the number of handovers that the call has already suffered. Therefore the average number of handovers per call attempt, $n_{n}$, results in: 


$$
n_{h}=\frac{\left(1-P_{b 1}\right) P_{h}}{1-\left(1-P_{b 2}\right) P_{h}}
$$

Where:

$$
\begin{aligned}
\text { - } \quad P_{b_{1}}= & \text { blocking probability for new call } \\
\text { attempts; } & \\
\text { - } \quad P_{b_{2}}= & \begin{array}{l}
\text { dropping probability due to } \\
\text { unsuccessful handover. }
\end{array}
\end{aligned}
$$

Hence, the time during which a call stays in a cell, can be defined as:

$$
t_{H} \Delta \min \left[t_{m c}, t_{0}\right]
$$

Recalling that $t_{d}$ is exponentially distributed, the p.d.f. of $t_{H}$ can be derived from (5) and (9). Therefore the expected value of $t_{H}$ is:

$$
E\left[t_{H}\right]=T_{m}\left(1-P_{h}\right)
$$

Equation (10) points out that the effect of handovers on the call permanence time in a cell is that its expected value is less than the expected call duration time, $T_{m}$.

In our study the handover arrivals process has been modeled as a Poisson process, independent of the new call arrivals process and with average arrival rate $\lambda_{h}$ related to $\lambda$ by:

$$
\left[\lambda\left(1-P_{b 1}\right)+\lambda_{h}\left(1-P_{b 2}\right)\right] P_{h}=\lambda_{h}
$$

Equation (11) has been derived by considering a stability condition which in our case means that the rate of mobile users leaving a cell is equal to the rate of mobile users entering the cell.

Finally, we note that from (11) and (8) we have:

$$
\lambda_{h} / \lambda=n_{h}
$$

\section{RESULTS AND COMPARISONS}

In this section the performance of the DCA technique in terms of call blocking probability is compared with the one we obtain by using a Fined Channel Aflocation (FCA) technique.

In a FCA technique a set of channels is permanently assigned to each cell. The same set of channels is re-used in another cell at a distance D away. The basic FCA technique implies that a call attempt at a cell site can only be served by an available channel belonging to the set of channels assigned to that cell sile; otherwise the call is blocked.

In our simulations we have considered a parallelogram-shaped cellular network, with $\mathrm{N}$ cells per side [2]. The results on the btocking probability have been gathered only from the central cells, i.e. the cells with a complete interfering belt. It is straightforward to note that these cells have the highest constraints number to be satisfied in performing the channel allocation, therefore resulting in the highest blocking probability values.

Fig. 2 shows the blocking probability, $P_{b}$, for the proposed DCA technique as a function of the traffic intensity (i.e. $\lambda T_{m}$ ) for each cell for the case $\alpha \rightarrow \infty$ (i.e. no handover). These simulation results have been obtained using the following system parameters:

- $\quad N=7$ (cells per side);

- $\quad$ set of interfering cells of $x$ formed by two tiers of adjacent cell of $x$ (i.e. a reuse of 7 );

- $\quad M=70$ (channels).

In the same figure $P_{b}$ achieved in the case of a FCA technique is also reported for comparison purposes. In particular, $P_{b}$ for the FCA technique can be theoretically derived by means of the ERLANG-B formula with 10 channels per cell.

Fig. 2 highlights the better performance of the DCA technique. These advantages are reduced under high traffic load conditions (network congestion [3]).

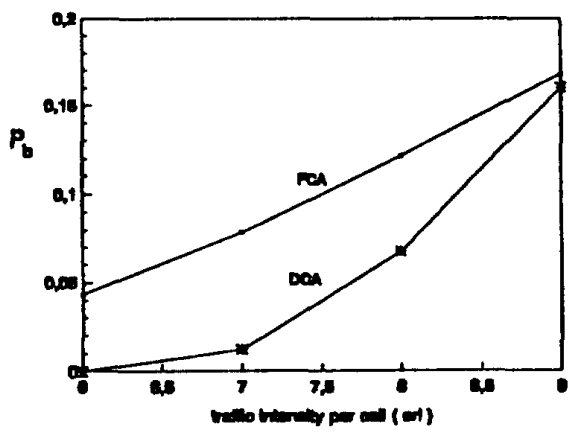

Fig. 2: comparison between DCA and FCA in the case of fixed users $(\alpha \rightarrow \infty)$.

Figg. 3 and 4 show the behaviours of the blocking probability (both for new call attempts and for handover attempts) as a function of the traffic 
intensity per cell, for DCA and FCA in two different mobility cases, i.e. $\alpha=0.6$ and $\alpha=0.35$ (typical for microcellutar systems). Also for these simulations the system parameler values are chosen equal to the ones used belore (fig. 2), i.e. $N=7$, interfering cells formed by two tiers and $M=70$.

The considerations we can get out from these figures are:

like in the case with fixed users $(\alpha \rightarrow \infty)$, the DCA lechnique outperiorms the FCA technique under medium and low traffic conditions; on the contrary under high traflic loed conditions advantages are reduced till we have a trend inversion. However it is important to point out that in such conditions of congestion, the values of the blocking probability are so high that the network will be never designed to operate in these situations.

If the users mobility increases (decreasing $\alpha$ ) the blocking probability decreases at the same traffic load conditions, both in the case of FCA and DCA techniques.

- The proposed DCA technique reduces the blocking probability of handover attempts with respect to the one of new call attempts by an efficient use of the dynamic channel de-allocation algorithm outlined in sect. II. An analogous advantage cannot be obtained by FCA technique, because the calls management in a cell is independent from what happens in the adjacent cells.

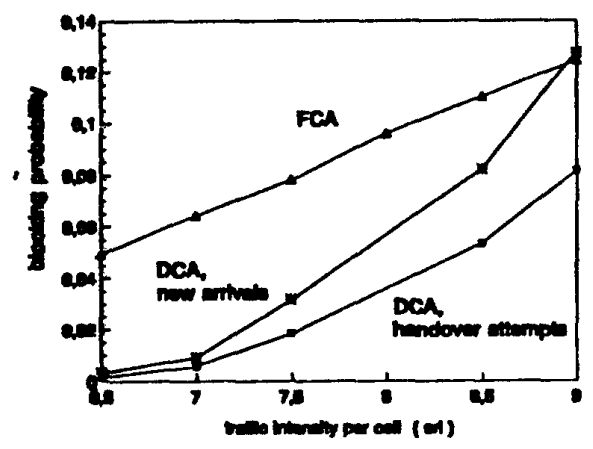

Fig. 3: comparison DCA - FCA for: $T_{m}=$ $1.76 \min , V_{\max }=100 \mathrm{Km} / \mathrm{h}, \mathrm{P}=0.88 \mathrm{Km} \Rightarrow \alpha=0.6$.

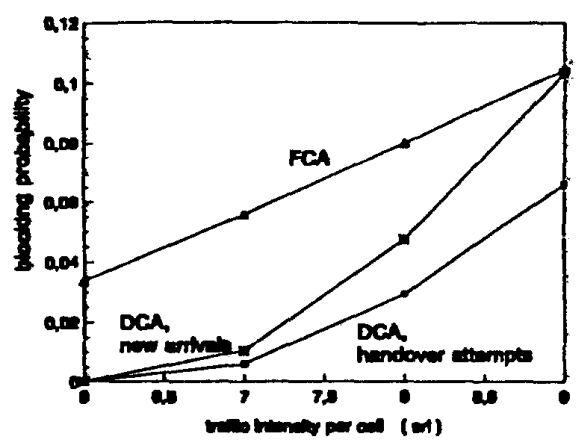

Fig. 4: comparison DCA - FCA for: $T_{m}=$ $3 \min , V_{\max }=100 \mathrm{Km} / \mathrm{R}=0.88 \mathrm{Km} \Rightarrow \alpha=0.35$.

\section{v. CONCLUSIONS}

In this paper an efficient DCA technique suitable for application in terrestrial and satellite mobile communication systems has been presented. This DCA technique makes use of a cost function defined for each available channel to achieve an optimum channel allocation on demand. In addition to this, a suitable channel de-allocation is periormed whenever a call termination occurs in a cell to increase the spectrum efficiency. Moreover it has been shown that the DCA technique permits to reduce the probability of unsuccessful handover. This is of remarkable importance to obtain a good quality of service when the number of handovers per call is high as in microcellular systems.

\section{REFERENCES}

[1] V.H. MacDonald, "The Cellular Concept," Bell Syst. Tech. J., vol. 58, pp. 15 - 41, Jan. 1979.

[2 ] M. Zhang, and Tak-Shing P. Yum, "Comparisons of Channel-Assignment Strategies in Cellular Mobile Telephone Systems," IEEE Trans. on Veh. Tech., vol. 38, n. 4, pp. 211 - 215, Nov. 1989.

[3] Paul - André Raymond, "Performance Analysis of Cellular Networks," IEEE Trans. on Commun., vol. 39, n. 12, pp. 1787 - 1793, Dec. 1991.

[4] S. Nanda, and D.J. Goodman, "Dynamic Resource Acquisition: Distributed Carrier Allocation for TDMA Cellular Systems," Proc. GLOBECOM '91, pp. 883 - 888, Phoenix, Arizona, 2 - 5 Dec. 1991.

[5] S. Tekinay, and B. Jabbari, "Handover and Channel Assignment in Mobile Cellular Networks," IEEE Communications Magazine, vol. 29, n. 11, pp. 42 - 46, Nov. 1991. 